\title{
Software tools for the quantitative evaluation of dental treatment effects from $\mu C T$ scans
}

\author{
R. Sinibaldi ${ }^{1}$, A. Conti ${ }^{1}$, R. Pecci ${ }^{2}$, G. Plotino ${ }^{3}$, R. Guidotti ${ }^{1}$, N.M. Grande ${ }^{3}$, M.G. Ortore ${ }^{4}$, C. Becce ${ }^{5}$, \\ R. Bedini ${ }^{2}$, S. Della Penna \\ 1. Department of Neuroscience and Imaging, University G. D'Annunzio of Chieti and Pescara, Chieti, Italy. 2. Technologies \\ and Health Department, I stituto Superiore di Sanità, Rome, I taly. 3. Department of Endodontics, "Sapienza" University of \\ Rome, Rome, I taly. 4. Department of Life and Environmental Sciences, Marche Polytechnic University, via Brecce Bianche, \\ Ancona, Italy. 5. Private practitioner in dentistry, Savona, Italy
}

Correspondence: Raffaele Sinibaldi. Address: Department of Neuroscience and Imaging, University G. D'Annunzio of Chieti and Pescara, via dei Vestini 31, 66100 Chieti, Italy. E-mail: r.sinibaldi@unich.it

Received: May 14, 2013

Accepted: June 17, 2013

Online Published: August 2, 2013

DOI : $10.5430 / j b g c . v 3 n 4 p 85$

URL: http://dx.doi.org/10.5430/jbgc.v3n4p85

\section{Abstract}

Background: The 3D images of dental specimens were obtained by means of micro-Computed Tomography $(\mu \mathrm{CT})$ before and after therapeutic intervention. A suite of software tools has been developed to assess the efficacy of dental treatment as revealed by $\mu \mathrm{CT}$ scans. Endodontic root canal and restorative therapy were selected as model procedures to test and optimize the developed tools. Non destructive $\mu \mathrm{CT}$ imaging allows repeated scans of the same tooth and might provide quantitative information about specimen modifications over time. Preliminary literature has suggested that anatomical characterization and detailed evaluation of dental therapies would considerably benefit from the coupling between detailed 3D imaging and custom software. However, the main drawbacks reside in time consuming pipeline for scanner-time of the specimens and absence of efficient software for accurate data analysis and direct comparison of a set of 3D dental structures.

Methods: The software we present here implements co-registration procedures together with an algorithm for the 3D quantitative characterization of teeth hollow spaces and interfaces. The software is targeted to the individualized analysis of the single specimen in an effort to help dentists to quantify the outcome of the surgical intervention performed on teeth. The approach aims to automatically highlight the differences between pre- and post-treatment structures for further anatomical and statistical analysis. Of note, the two different treatments analyzed exploit two different perspectives. As a matter of facts, endodontic therapy aims at the modification of the root canal structure (axe, volume and surface), whereas the restorative treatment focus is on the whole tooth structure.

Results: The developed co-registration algorithm allows finding correct superposition in both data sets, despite the preand post-structure can be markedly different, especially after the application of dental adhesive composite in the restorative treatment. Tools for characterization of canal volume and surface are robust with respect to the presence of branches and allow the complete characterization of the endodontic treatments. Modification of the tooth structure after restorative treatments is highlighted after the co-registration procedure in automatic way and allows the user to quantify the volume of voids due to the shrinkage of the material at the interface and eventually the presence of internal cracks or other morphological modification of the tooth structure. 
Conclusions: We demonstrate the effectiveness of customization of 3D data analysis tools to obtain specific characterization and quantitative information about different dental therapies.

\section{Key words}

Micro-computed tomography, Dentistry, Software tools, Co-registration, Quantitative evaluation of canal anatomy

\section{Introduction}

Micro-computed tomography $(\mu \mathrm{CT})$ is becoming a mature technology with promising applications in the study of hard tissues with a special attention to dentistry. Nowadays, laboratory $\mu \mathrm{CT}$ instruments can produce images with isotropic voxel side smaller than $10 \mu \mathrm{m}$. This high spatial resolution is adequate for the reconstruction of small objects such as teeth $(\sim$ few $\mathrm{cm})$, trabecular bone structure $(\sim$ some $\mathrm{mm})$ and newly formed tissue around dental implants $(\sim$ few tens of $\mu \mathrm{m})$.

$\mu \mathrm{CT}$ proved to be a valuable method in different research contexts in dentistry such as endodontics ${ }^{[1,2]}$, dental restoration ${ }^{[3,4]}$ and materials for maxillofacial implant surgery ${ }^{[5,6]}$. This technique allows obtaining high quality $3 \mathrm{D}$ images of specimens, which can be compared with histological evaluations ${ }^{[7,8]}$. Thanks to its intrinsic non-destructive character, $\mu \mathrm{CT}$ can be used to provide evidence of tissue surface and volume changes over time. As an example, it is possible to scan a sample before and after a specific treatment, in order to evaluate possible modifications of its 3D structure ${ }^{[1,2,9]}$. Furthermore, internal and external anatomy can be provided simultaneously or separately ${ }^{[10]}$. The main drawback is that $\mu \mathrm{CT}$ is not suitable for clinical use, because a high X-ray dose is required for micrometer resolution. However cone-beam computed tomography (CBCT) has been recently introduced for imaging hard tissues of the maxillofacial region ${ }^{[11,12]}$ and it is now often used in the planning of intervention and in patient follow-up, opening possibility for quantitative evaluation of $3 \mathrm{D}$ dental data for the patient specific health care ${ }^{[13,14]}$.

The use of a tailored software in combination with tomographic techniques gives rise to a virtual laboratory ${ }^{[15,16]}$, able to investigate $3 \mathrm{D}$ structures, to characterize homogeneity and interfaces ${ }^{[17,18]}$, to predict permeability in porous media and hence accessibility of drugs and cells in different tissues ${ }^{[19]}$. It is becoming more and more common to evaluate physical properties via numerical simulation starting from the real $3 \mathrm{D}$ structure of the specimen revealed by $\mu \mathrm{CT}$, to access micro-mechanic features and failure behavior of materials and composites under simulated stress conditions ${ }^{[20-23]}$.

In this context there is an increasing demand of robust and user-friendly software tools, which can provide quantitative evaluation of different parameters from the 3D reconstructed structure of a tooth. In the field of dentistry the lack of this software limits the spread of $\mu \mathrm{CT}$ even more than the instrumental availability ${ }^{[1,2,24]}$. Characterization of dental treatment can require several software tools, which, even if available for other applications, are far from being standardized and targeted to the research in dentistry ${ }^{[7,25]}$. As an example, evaluation of endodontic therapy success requires that the 3D reconstruction of the tooth structure detects possible differences as revealed by $\mu \mathrm{CT}$ before and after modification of the healed site. Quantitative information is extracted from $\mu \mathrm{CT}$ data by means of direct comparison between the surface of the canals before and after treatment in a common reference system ${ }^{[2,9,26]}$. After endodontic treatment, it is possible to observe the overlapped pre- and post-treatment $3 \mathrm{D}$ structures and detect if there are zones of the canal which are untouched.

We present here a software package, including different tools, useful to obtain quantitative evaluation of different parameters characterizing the 3D morphology of a tooth and root canal system. We apply this software to assess the buoyancy of two different therapeutic procedures: endodontic and dental conservative treatment. The software package includes algorithms for quantitative analysis of endodontic therapy, such as the estimation of the percentage of treated canal surface (PTCS), the volume of dentin removed by mechanical preparation, and the modification of the root canal axis in the 3D space. Additionally, a tailored algorithm has been developed to compare the $\mu \mathrm{CT}$ structure of a tooth, with 
preparing cavities, before and after restoration with adhesive photopolymer. The aim is here at evaluating the quality of the cohesive bond and whether the composite material could modify the original tooth structure during the shrinkage effect.

We present the analysis of $\mu \mathrm{CT} 3 \mathrm{D}$ structures of two different teeth, one molar and one incisive before and after endodontic and conservative treatments respectively, and we apply different algorithms to compare quantitative morphological parameters to evaluate the buoyancy of the two different therapies. The analysis of these two different examples is of general interest in dental research since they are dealing with characterization of the bulk tooth structure and with the detailed canal anatomy.

\section{Subjects and methods}

\subsection{Endodontic treatment}

One maxillary first molar was scanned by $\mu \mathrm{CT}$. This sample was selected from a pool of extracted teeth from an Italian population (age ranging from 35 years to 55 years). After extraction, the tooth was cleaned in $5 \% \mathrm{NaOCl}$ solution for $24 \mathrm{~h}$, debrided of periodontal tissue and calculus, washed under running water, blotted dry and stored in saline solution. The criteria for selection were the following: fully formed apices, no restorations with intact crowns and no defects or carious lesions.

Preoperative radiograph of the tooth was exposed using a paralleling technique to determine approximate root canal lengths. The radiograph was also used to detect calcified root canals to be excluded from the study. After that access openings were prepared, the canal orifices were located and the cavity irrigated with $5.25 \% \mathrm{NaOCl}$. The canals were initially scouted with sizes 08 and 10 hand flexible K-type files using a lubricant. The canal working length was determined and then confirmed radiographically.

Root canal treatment was performed on extracted tooth using a standard clinical protocol by means of ProFile Ni-Ti rotary instruments. The ProFile system consists of several instruments varying in tip size and taper. The clinical sequence used was: orifice shaper number 3 and 2 to enlarge the orifice, a crown-down root canal preparation using size 25, 0.06 taper, size 20, 0.06 taper, size 25, 0.04 taper, size 20, 0.04 taper, to prepare the canal and size 20, 0.06 taper and size 25, 0.06 taper to refine the apex. The ProFile Ni-Ti rotary instruments were used at a constant speed of $300 \mathrm{rpm}$ in a 6:1 reduction hand-piece powered by a torque-controlled motor. The patency of the apical foramen was checked by passing the tip of a size 08 file through the foramen after each instrument of the sequence until completion of the root canal shaping. During shaping, each canal was irrigated between each successive instrument with $2.5 \mathrm{ml}$ of $5.25 \% \mathrm{NaOCl}$ using an endodontic syringe placed as far into the root canal as possible without binding. The final flush was performed with $5 \mathrm{ml}$ of $17 \%$ EDTA solution rinsed out with $5 \mathrm{ml}$ of saline solution. Canals were then dried with paper points. Each instrument was carefully examined under the stereomicroscope at 10× magnification (Kaps, Aalen, Germany) between uses for signs of plastic deformation or separation and excluded from the study if any damage was present.

\subsection{Conservative treatment}

One human incisive was extracted from a patient (30 year old) suffering for dysodontiasis and stored in physiological solution. A cavity was prepared in the central portion of the occlusal surface to simulate tooth restoration, and then measured and evaluated by means of $\mu \mathrm{CT}$. Tooth cavity dimension was computed on four orthogonal cross-sections and in three points along each dimension. The dimensional mean values of the created cavity were $6 \mathrm{~mm}$ height and $5 \mathrm{~mm}$ diameter. To avoid angle formation, the cavity was prepared using a round diamond bur with $2.5 \mathrm{~mm}$ diameter. The cavity was cleaned with air-water spray and softly dried to ensure wet surface. After etching dental adhesive was applied, according to manufacturer's operating manual, to facilitate bonding to the tooth substance and composite material was used to restore the portion of tooth removed. 
The selected adhesive system was applied according to manufacturer's guidelines and the cavity was filled. Increasing layer's polymerization ( $2 \mathrm{~mm}$ maximum thickness) of the composite has been applied for a period of 45 seconds for each layer, using a LED rechargeable blue lamp (mod. 655/00, De Giorgi, Italy) with $420 \mathrm{~nm}-480 \mathrm{~nm}$ wavelength, then the tooth was stored in physiological solution for $48 \mathrm{~h}$ until it was subjected to second $\mu \mathrm{CT}$ scan.

\section{$2.3 \mu \mathrm{CT}$ experiment}

The sample holder of the $\mu \mathrm{CT}$ was selected according to the specimen size and the necessary magnification range; throughout our study, a sample holder with a diameter of $15 \mathrm{~mm}$ was used. A custom attachment made by acrylic resin has been fabricated for each tooth to exactly fit the specimen and the specimen holder of the $\mu \mathrm{CT}$ apparatus. This attachment allowed repositioning of the specimen in the scanning system along the z-axis (error $\approx 400 \mu \mathrm{m}$ ) with minimal rotational error $\left(\approx 5^{\circ}\right)$. The $\mu \mathrm{CT}$ acquisition procedure of each sample consisted of two stages requiring approximately four hours in all: two hours for the scanning and two hours for the reconstruction procedure.

A micro-computed tomography scanner (SkyScan 1072, Aartselaar, Belgium) was used to analyse the specimens, which were scanned before and after dental treatment. The scanning procedure used $10 \mathrm{Watts}, 100 \mathrm{kV}, 98 \mu \mathrm{A}, 1.0 \mathrm{~mm}$ aluminum filter and $\times 15$ magnification, resulting in a pixel size of $20 \times 20 \mu \mathrm{m}^{2}$. Hundreds of projections were acquired through 180 degrees of rotation and then transformed into a stack of about 750 new 2D images. Each image is an axial cross-section composed by $1,024 \times 1,024$ voxels, 8 bit grey scale, with $20 \times 20 \mu \mathrm{m}^{2}$ in-plane resolution and a slice thickness of $40 \mu \mathrm{m}$. Further details about the scanning and reconstruction procedure can be found elsewhere ${ }^{[10,27]}$.

\subsection{I mage pre-processing}

The $\mu \mathrm{CT}$ images are preliminary segmented to distinguish between different materials phases. Evaluation of endodontic therapy requires detection of the void spaces inside the tooth; hence the segmentation deals with only two phases. For the characterization of conservative therapy three phases have been considered: native tooth structure, adhesive composite material and eventually voids. The segmentation process needs high accuracy, especially to characterize the interfaces between different materials composing the restored tooth structure ${ }^{[28,29]}$. In the reconstructed $\mu \mathrm{CT}$ images the dental adhesive appears as a more radio-opaque material, native tooth has lower absorption and voids and soft tissue are almost transparent to X-ray. As a consequence of the co-registration procedure (see below) there is no need to distinguish between composite material and native tooth, hence only one threshold is applied to distinguish between solid materials and voids. Specifically, both teeth are segmented considering voxels with grey values lower than a chosen threshold to be part of the tooth. Moreover, also voxels with a value $10 \%$ higher than the chosen threshold, provided that they are first neighbors of voxels that are below the threshold are included in the tooth structure.

3D rendering of the teeth structures is produced using Amira 4.1 software (Mercury Computer Systems, Berlin, Germany) allowing contemporary visualization of more than one 3D dataset. The transparency effect of Amira has been used to visualize internal tooth features embedded inside the structures.

\subsection{Co-registration procedure - endodontic case}

The pre and post-treatment $3 \mathrm{D}$ tomographic reconstructions evidence a mismatch due to the inability to relocate the sample inside the sample holder with an accuracy higher than the single voxel resolution (see Figure 1). Evaluation of changes in the 3D morphology of the specimens requires local comparison of tiny anatomical structures such as root canal surface and interface between tooth and adhesive composite. The co-registration of data related to endodontic treatment is simpler with respect to conservative case because only the canals differ between pre and post treatment, hence the whole $3 \mathrm{D}$ tooth structure is preserved. The algorithm developed for this purpose is analytical and based on classical mechanics of rigid bodies. The first step is to virtually fill all the canals in both tooth structures, leading the tooth to become a 3D volume filled by homogeneous material. The pre- and post-treatment structures, after in silico canal filling, result to be identical. Also the inertia ellipsoids in the reference system of their own centre of mass are identical. After a rigid translation of the 
post structure to superimpose the two centres of mass, the algorithm calculates the inertia tensor and then transforms both tensors into diagonal form by means of Jacobi transformation method. This procedure provides two sets of 3D vectors, the free axis of rotation of the pre- and post-treatment structures, which are two independent orthonormal basis of the 3D space.
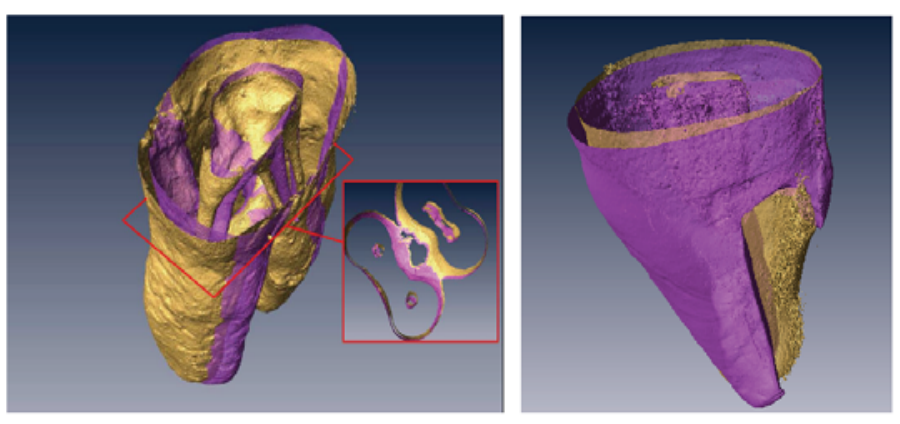

Figure 1. "3D rendering of original $\mu \mathrm{CT}$ data". Left side: Molar tooth, before (violet) and after (gold) endodontic treatment. In the inset, 3D reconstruction of few slices highlights the mismatch between canal surfaces. Right side: incisive tooth before (violet) and after (gold) application of the dental restorative material. 3D structures are visualization of original $\mu \mathrm{CT}$ and are not co-registered.

The sequence of three rotations necessary to superimpose the two sets of vectors can be calculated analytically and the same sequence of rotations is applied to obtain the superposition of the pre- and post-treatment structures ${ }^{[30]}$. The software provides one new stack of reoriented slices of the post-structure to obtain the desired superposition between the two. In particular, if the spatial resolutions of the two scans are different, the software creates the output slices using the worse resolution.

When applying rotation sequences, numerical truncation might generate voxels with undetermined values in the rotated body, due to overlap of multiple rotated voxels into a single new voxel. To overcome this issue, we applied the inverse of the rotation matrix within a loop running over each voxel of the pre-structure, which remains fixed. This forces associating all the voxels of the pre-structure with the voxel values in the post-structure addressed by the inverse rotation matrix. Also, the size of the rotated image is always larger than the original one, with the maximum being at $45^{\circ}$ rotation. Digital images are always of rectangular shape; hence the memory needed for structure allocation is $\sqrt{ } 2$ times bigger for each dimension with respect to the original one.

\subsection{Co-registration procedure - conservative case}

The analytical co-registration procedure described above cannot be applied to the dataset from the restorative therapy. The reason can be understood by inspecting the two structures to be superimposed Figure 1. The first $\mu \mathrm{CT}$ acquisition (coloured in violet) shows the incisive tooth after a cavity has been created to simulate caries removal. The second acquisition (coloured in gold) was performed after adhesive composite was applied and the tooth cavity was filled. The shape of these two 3D structures is not the same, their inertia ellipsoids are different, and hence the analytical algorithms based on classical mechanics of rigid bodies cannot be applied. Bergmans and co-workers ${ }^{[2]}$ used a minimization approach to find the best superposition between two scans of the same tooth for the evaluation of endodontic therapy. The procedure maximizes the Mutual Information ${ }^{[31]}$, a functional based on the information that one variable contains about another variable. Mutual Information estimated on intensity values of corresponding voxel pairs is maximal if the images are geometrically aligned. In our approach based on Mutual Information, we applied the Adaptive Simulated Annealing (ASA) ${ }^{[32]}$ as minimization algorithm. The cost function employed is the Normalized Mutual Information (NMI), defined in Eq. (1), which has been demonstrated to be less sensitive to the size of the overlapping part of the two 3D structures than Mutual Information ${ }^{[33]}$. 


$$
N M I(A, B)=\frac{H(A)+H(B)}{H(A, B)}
$$

The NMI calculated using two images A and B, NMI(A,B), depends on the function $H(X)$, which is the Shannon entropy of image $X$, and on $H(A, B)$, which is the joint entropy of images $A$ and $B$. The definition of image entropy is related to information theory ${ }^{[34]}$ and can be found elsewhere ${ }^{[35,36]}$. NMI can then be used also if one of the two dataset contains only a portion of the entire 3D structure.

When dealing with restorative treatment, which implies the application of adhesive composite on the incisive tooth, we cannot assume the structure variations to be small changes inside wider, overlapped structure as in the endodontic treatment. It is clear from Figure 1 and the discussion above that the differences involve a rather big volume fraction of the two samples and this considerably increases the computational resources and time needed by the minimization algorithm. Indeed, minimization algorithms usually adopted in the literature start from an initial set of parameters chosen by the user, and this determines the best solution achieved. The algorithms are launched many times from different initial guesses, making these strategies time consuming and user dependent. To overcome this issue and to exploit the potentiality of the NMI as a cost function reducing the needs of high computational resources, we adopted the following strategy: the user is allowed to choose several (minimum three up to 100) Regions Of Interest (ROI) in the 3D space. These ROIs are selected only on the pre-treatment 3D structure and possibly far from the treated volumes, and are then processed by the algorithm instead of the whole tooth volume. The algorithm looks for the correct superposition between pre- and post-structure by calculating the NMI between the selected ROIs and the corresponding inverse rotated ROIs on the post-structure (as explained previously). We adopted parallelepiped ROIs to simplify the selection procedure, but they could be an arbitrary 3D shape. We found it efficient to place some ROIs near interfaces between the tooth structure and a void. Indeed these specific ROIs drive the minimization algorithm since the cost function increases considerably also if the mismatch is rather small due to wrong overlaps between material and background.

Although this procedure is not analytical and requires user input, it results to be very efficient because the computational resources depend on the volume of the selected ROIs (total number of voxels belonging to the ROIs) and not on the entire volume of the specimen. Additionally, the first guess involves about $1 \%$ of the total volume, reducing of two order of magnitude both memory resources and computational time with respect to the total volume.

\subsection{Quantitative endodontic analysis}

An algorithm quantifying the volume of dentin removed and PTCS has been developed. At this aim, the pre- and posttreatment tooth canal structures are simultaneously processed. The algorithm allows the user to choose the canal path under study in presence of anatomical bifurcations. The output comprises a new set of slices containing the detected shape of both pre- and post-treatment canal cross-sections after co-registration. Removed volume of dentin and PTCS data are calculated for each slice as a function of canal curvilinear abscissa.

The canal is divided into three sections and the mean value of the PTCS and total volume of dentin removed for each canal section (apical, middle and coronal) are calculated by the software to evaluate the efficiency of the endodontic treatment, as suggested in previous literature ${ }^{[2,9,26]}$. PTCS error bars are evaluated for each canal section as the standard deviation of the PTCS data calculated over all the tomographic slices included in each canal section. Error bars of the volume ( $\Delta S$ ) of dentin removed are evaluated summing the errors on the estimation of the canal section areas $(\Delta S)$ over the length of the selected canal section. Approximating the canal section area to a circle, the canal surface enlargement can be assumed as a ring. Being $r$ and $R$ the pre and post treatment radius of the assumed canal circle, the error on the estimation of the enlarged ring surface is: $\Delta S=2 \pi\left(R^{2}-r^{2}\right) \Delta R / \bar{R}$. Where $\Delta R$ is the voxel side length and $\bar{R}$ is the mean value between $r$ and $R$. 


\section{Results}

The results obtained with the two co-registration methods are reported in Figure 2. In Figure 2(A) the 3D tooth structures acquired before and after endodontic treatment are aligned. The inset of Figure 2(A) contains a 3D rendering of few slices. In these sample slices, the effect of endodontic treatment on the canal surface is shown and we can observe that some zones of the canal surface are not modified by the treatment and the pre- and post- canal surfaces result to be superimposed. In Figure 2(B) we report the 3D structure of the incisive tooth after cavity preparation but before the application of the adhesive composite. Figure $2(\mathrm{C})$ is the result of the co-registration obtained maximizing the NMI calculated on 12 ROIs distributed through the whole native incisive volume acquired before application of the polymer composite. The 12 ROIs were made by approximately 950,000 voxels, which is less than the total number of voxel contained in a single $\mu \mathrm{CT}$ slice. The pictures in Figure 2(C) represent the 3D rendering of the superposition between the $\mu \mathrm{CT}$ acquisition of the incisive before and after application of the composite material. White represents the incisive native structure, green the adhesive composite, in red we show the structural modifications due to the effect of composite shrinking as explained in the following.
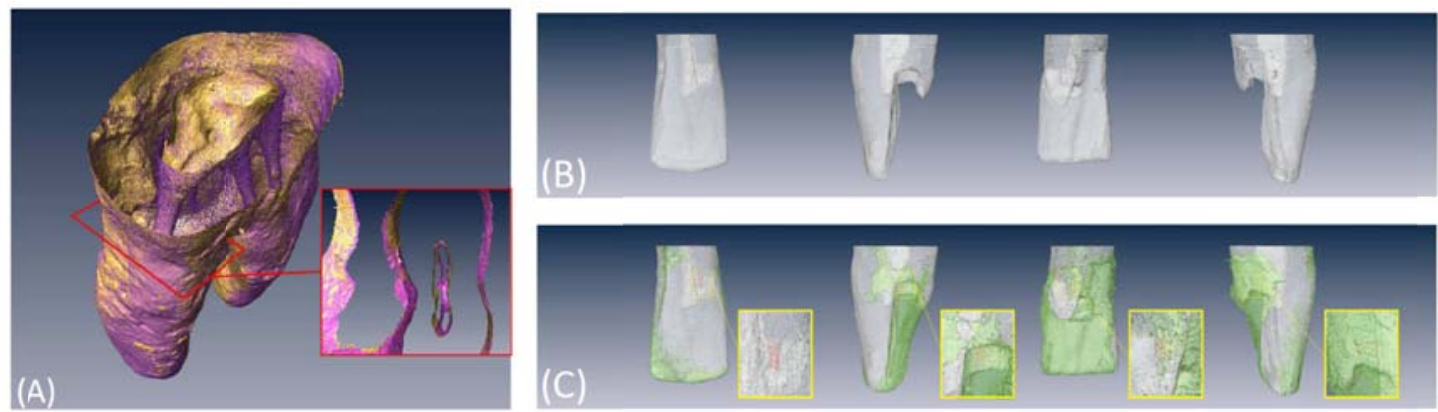

Figure 2. "Co-registered data". A) Co-registered images of pre and post treatment molar tooth. B) Pre structure in white before application of adhesive composite and C) after restorative treatment. Adhesive composite is in green; red zones are part of the native tooth where pre- and post-structures are different.

\subsection{Characterization of endodontic treatment}

For the endodontic case an algorithm has been developed with the aim at analyzing the canals inside the tooth and trace the curvilinear abscissa of the canal axe along its path. Firstly, the user selects one of the tooth canals, and then the algorithm simultaneously processes the pre- and post- structure. Specifically, the algorithm looks for the canal in both the two co-registered structures and calculates the pattern of the canal surfaces shown in Figure 3. Eventually, the pre- and post-treatment corresponding canals are depicted in a common reference system and the enlargement of the canal cross-sections, along its curvilinear abscissa, can be visualized. The changes in the canal surface after endodontic treatment are sometimes minimal and then neglected, while for other canals the changes could be considerable.

If we browse through the canal from the crown to the apex extracting slices as the ones reported in Figure 3, the incremental quantity of dentin removed at each slide together with the percentage of treated canal surface can be estimated (see Figure 4). This allows evaluating the quality of the treatment, which is associated with uniform modification of the original canal surface. Specifically, when the canal enlargement is not uniform or the PTCS is low, the quality of the treatment was poor and the cleaning procedure of the canal failed. Quantitative data on the volume of dentin removed and PTCS in the treated canals as a function of curvilinear abscissa are reported in Figure 4. In Figure 5 we show the whole tooth structure composed by three roots and one canal for each root, where the red zones are the ones where the pre- and post-treatment canal surface overlap and hence the quality of the treatment is not satisfactory. The lower treatment efficiency is located in zones where the canal curvature changes i.e. zones pointed by white arrows in snapshot (a) and (d) of Figure 5. The software recognizes untreated surface zones and then it is possible to calculate correlations between the 
buoyancy of the treatment and the pattern of the canal axe. The position of the canal centre in 3D (quantitative data not shown, qualitative data shown in Figure 5) together with the quantitative data in Figure 4 allow to correlate the volume of removed dentin and the PTCS with the presence of curves, constrictions, and bifurcations. This set of tools can then be used to correlate the presence of critical canal features with the cleaning ability of the used endodontic instruments.
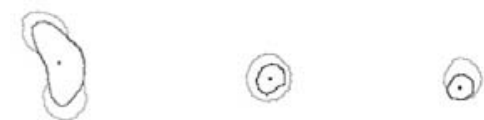

(.)
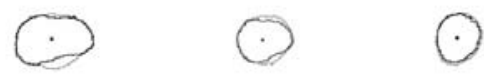

(e)

Figure 3. "Pre- and post-treatment canal surfaces". Four cross-sections of the three canals surface of the analysed tooth. The pre-treatment canal is black, the post-treatment canal is grey.
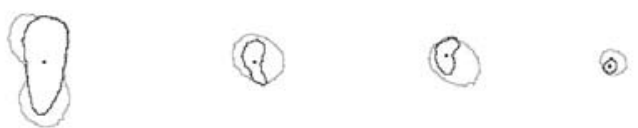

(a)
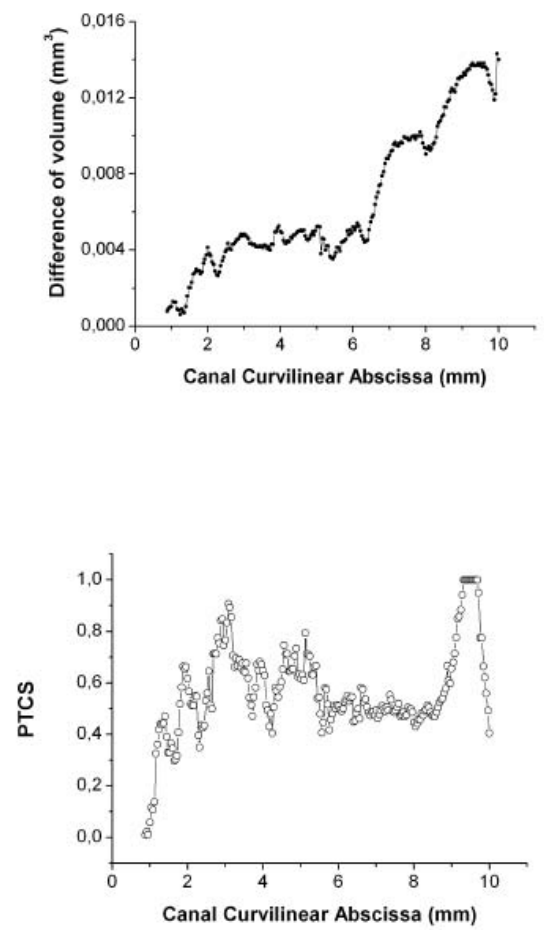

(b)
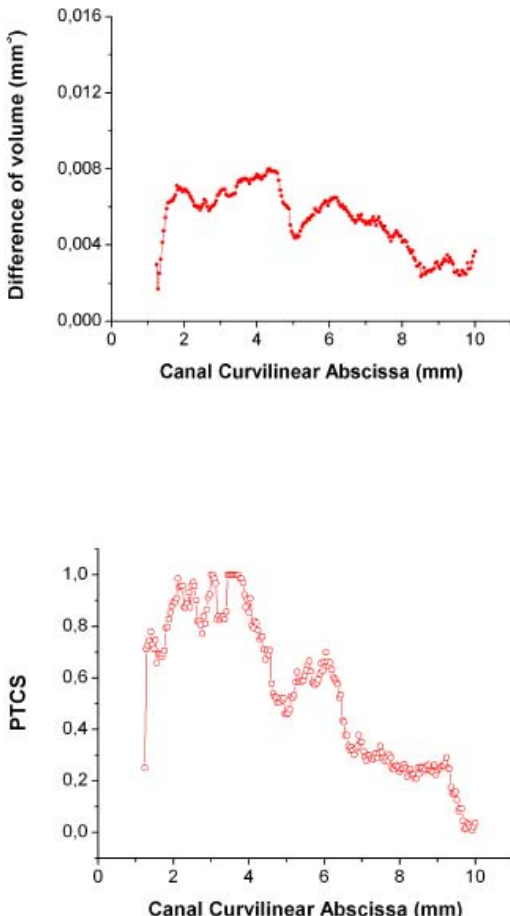

(c)
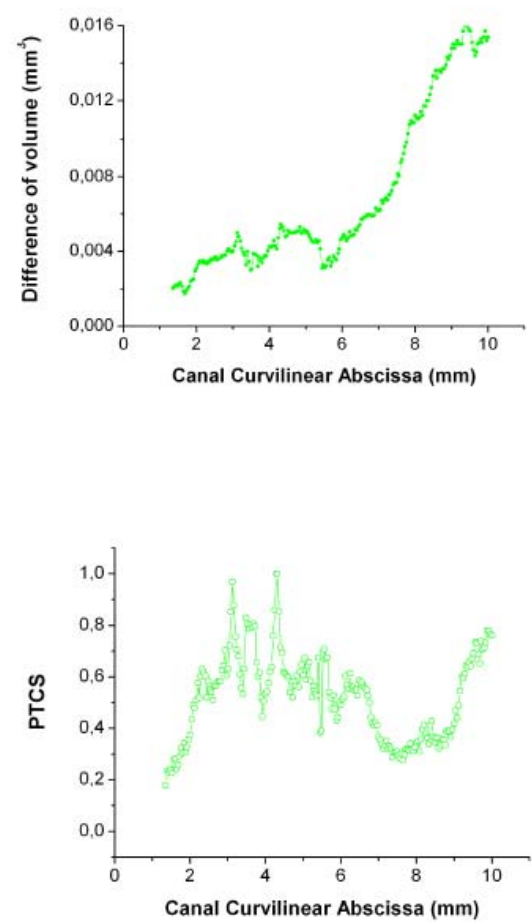

Figure 4. "Quantitative canal evaluation". Upper strip: difference of canal volume after and before endodontic treatment. Lower strip: percentage of touched canal surface (PTCS). Data are calculated every $40 \mu \mathrm{m}$ starting from the canal apex, on slices placed perpendicularly to the pre-treatment canal axe. 

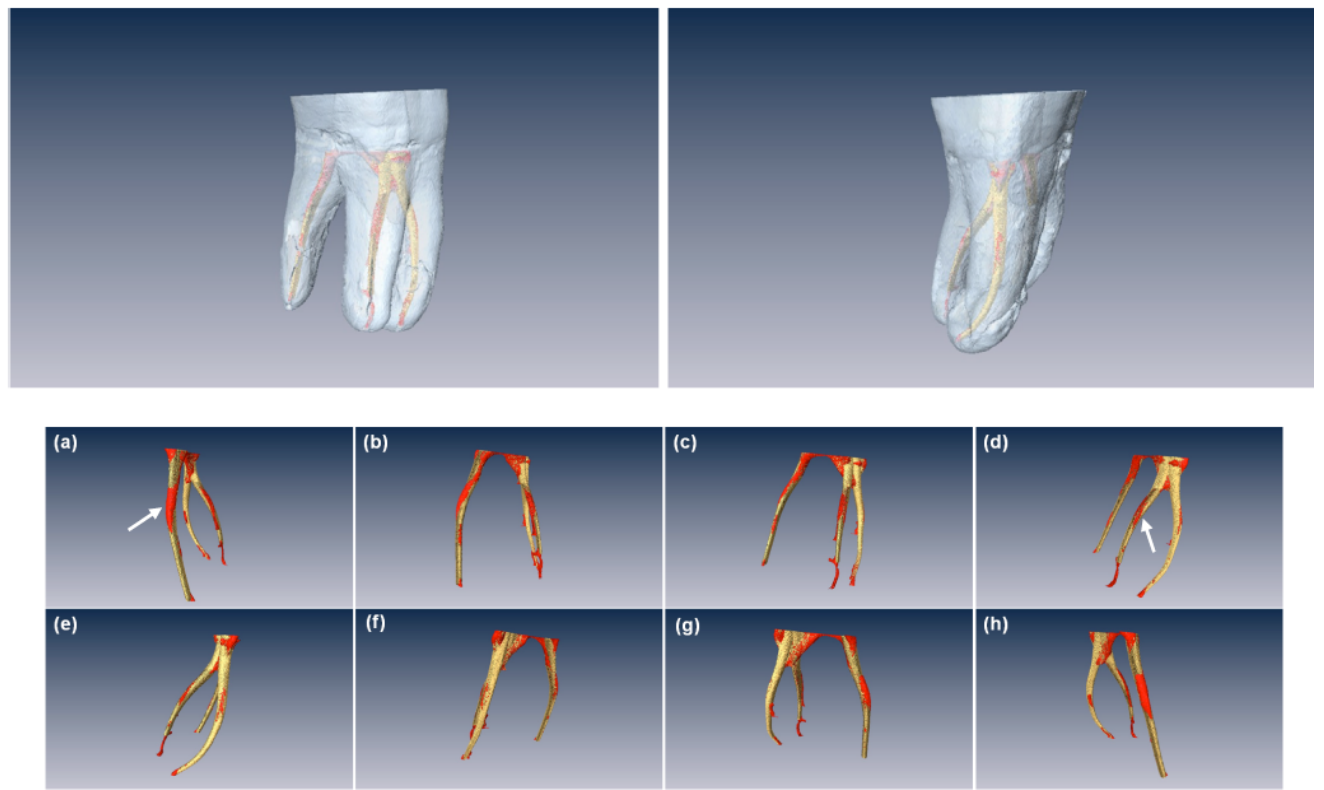

Figure 5. "3D structure of canals". Upper frame: tooth structure in transparency containing the canals. Lower frame: superposition of the root canals before and after treatment in the 3D space. Red means that pre and post canal surfaces are coincident, gold that the post canal surface is wider. Each snapshot correspond to $45^{\circ}$ rotation.

\subsection{Characterization of conservative treatment}

Two scans were performed on the incisive tooth: the first scan was performed after the preparation cavity had been created and the second scan was performed after the application of the adhesive composite. The 3D structure of the incisive, before co-registration, is shown in Figure 1. In Figure 2 the pre-structure, before application of adhesive composite (upper raw), is depicted in white. The lower row shows the co-registration between the native tooth, with the cavity, and the tooth after conservative treatment. The adhesive composite is displayed in green and red zones are volume portions inside the native structure, which have been modified during the shrinkage of the composite.

It is well known ${ }^{[4,37]}$ that, due to the shrinkage, manual filling of the cavity with the adhesive material is not perfect in the sense that void spaces are often found at the interface with dentin. It is of fundamental importance to analyze the outcome of the filling, since this procedure can compromise the stability of the new structure (teeth plus adhesive material), and increases the possibilities of material degradation. In Figure 6 two cross-sections of the 3D restored incisive are reported. These pictures have been obtained after the co-registration of the pre- and post- structure applying an algorithm able to distinguish between four different cases. When the two corresponding voxels of the pre- and post-treatment structure are both below the threshold the voxel in the co-registered structure belongs to native tooth (black), when both voxels are above the threshold, the co-registered voxel belongs to void space (white). The dark grey voxels in Figure 6 correspond to voxels that are above the threshold in the pre-structure (void space) and below in the post-structure (applied composite material), and light grey is vice versa (native in pre-structure and void in post-). Light grey means that the void spaces, or defects, are created by the application of the composite material and are somehow related to the shrinking. The images shown in Figure 6 provide the evidence for the presence of an internal crack after the application of the adhesive material. The crack is in the micrometer scale: about $2 \mathrm{~mm}$ length, $600 \mu \mathrm{m}$ width and $800 \mu \mathrm{m}$ depth. Different colours adopted in Figure 6 are not obtained through segmentation of the post structure, but by a comparison of corresponding voxel grey values of the two co-registered structures. Voids inside the composite or at the interface between dentin and composite are white; there are also new voids near interface (light grey), which are not cracks. These voids correspond to fragments of dentin, which were pried from the native structure during shrinkage. The new stack of slices produced by the co-registration software is used to depict the detail in Figure 6 and the whole 3D structure in Figure 2 (bottom stripe). 
These co-registered representations are not prone to segmentation uncertainty and allow quantifying the effect of mechanical stress induced by the shrinkage of the adhesive material.

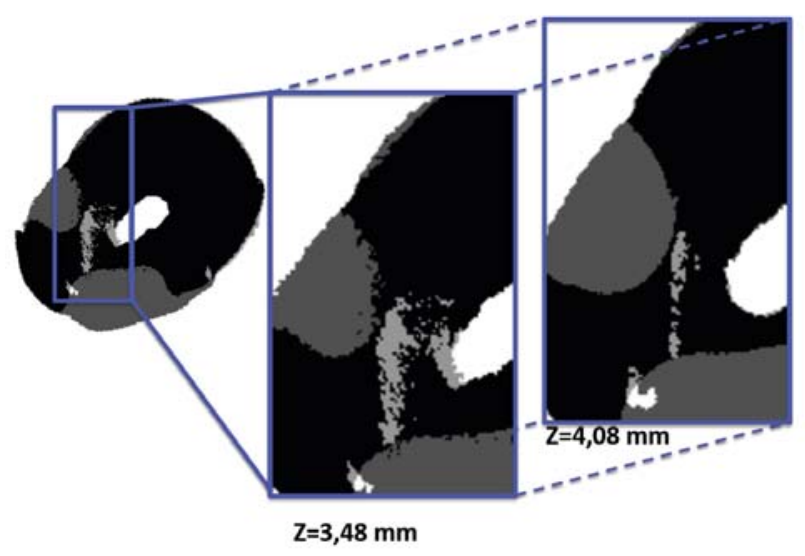

Figure 6. "Evidencing micro-crack by 3D co-registration". Two cross-sections of the 3D restored incisive structure. Black areas correspond to voxel below threshold in pre- and post- structures. The co-registered voxel is white when it is above threshold both in pre- and in post- structures. Dark grey pixels represent voxels above threshold in the pre-structure and below threshold in the post-structure, corresponding to areas where adhesive material was applied. Light grey pixels are the opposite and highlight the presence of an internal crack due to shrinkage of the adhesive material.

\section{Discussion}

In this pilot study a set of algorithms for the $3 \mathrm{D}$ quantitative analysis of $\mu \mathrm{CT}$ images have been applied to characterize tooth microstructures changes occurring after dental treatment.

The algorithms were designed to provide quantitative characterization of changes in root canal anatomy and tooth morphology. Two different co-registration approaches were developed to reduce computational time tailoring them on the different sample features. Quantitative parameters such as root canal volume and canal surface can be evaluated routinely. Software output stack of 3D images has been customized to highlight different anatomical changes occurring after sample treatment.

The whole set of algorithms was tested on two typical dental treatments, endodontic and restorative.

The analysis of the $\mu \mathrm{CT}$ dataset acquired on a premolar and an incisive promotes the need of pre- and post-therapy $\mu \mathrm{CT}$ experimental acquisitions.

The overlap of the images acquired before and after treatment provides valuable information about correlation between the curvilinear abscissa of the canal and the buoyancy of the endodontic treatments. Of note, unexpected results regarding micro-crack formation in the native tooth structure after photopolymerization shrinkage of the adhesive composite were highlighted by the presented software tools.

\subsection{Endodontic treatment}

The most important action to be taken in endodontic therapy is the tooth canal preparation, since proper preparation determines the efficiency of subsequent procedures. Root canal preparation includes mechanical debridement and creation of optimal canal geometries for medicaments delivery and filling ${ }^{[38]}$. Removal of debris by mechanical instrumentation aims at eliminating pulp tissue and microorganisms from the canal system. Cause of endodontic failure is the inability to 
debride and fill all canals ${ }^{[39,40]}$. Hence a deeper knowledge of the root canal system, patient specific variations and instrument performance in different context is a need for high quality canal treatment ${ }^{[41,42]}$.

In this pilot study we estimate quantitative parameters describing the endodontic outcome.

Traditional methods for the study of root canal morphology and its variations after different treatments include sectioning, clarifying techniques, traditional microscopy, SEM analysis and many others. All of them are destructive evaluations of the samples. It has been demonstrated that the quality of $\mu \mathrm{CT}$ outcomes is similar to the traditional techniques ${ }^{[43]}$. The significant advantages of $\mu \mathrm{CT}$ are the possibility of $3 \mathrm{D}$ imaging and the non-destructive evaluation, allowing to analyse multiple step treatments and to perform further investigation on the same specimen. The use of modern software is essential to exploit the potentiality of $\mu \mathrm{CT} 3 \mathrm{D}$ data. In particular co-registration is essential to obtain quantitative evaluation of endodontic treatment. Following the development of previous software and methods targeted to endodontic investigation ${ }^{[1,2]}$, we were able to increase reliability and flexibility of the analysis. Of course our analytical procedures are not general but suited for the endodontic case.

The software package described here overcomes technical difficulties in obtaining quick superimposition of the images before and after treatment. Great advantage has been achieved evaluating pre- and post-treatment structures in a parallel way, estimating the volume of the removed dentine and PTCS after cleaning and shaping. Using the software output, the quality of an endodontic treatment could be highlighted into the 3D space, depicting the effect of the instruments on the root canal walls or root-filling residue (see Figure 3 and Figure 5). The software produces a summary of quantitative evaluation of the endodontic treatment (see Figure 7). This summary is organized by the user himself, for example PTCS and removed dentine volume can be evaluated for each $\mu \mathrm{CT}$ section, for each millimeter of the canal length, for the three canal levels (apical, middle and coronal), or arbitrarily. The summary contains also the errors associated to the estimated parameters on the basis of the user choice.

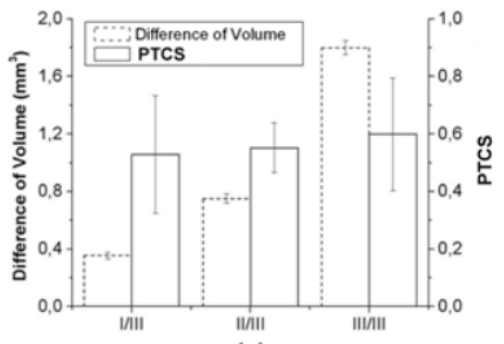

(a)

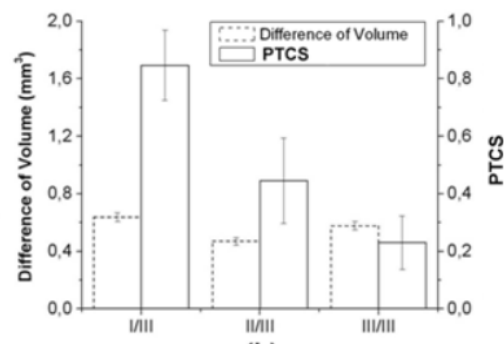

(b)

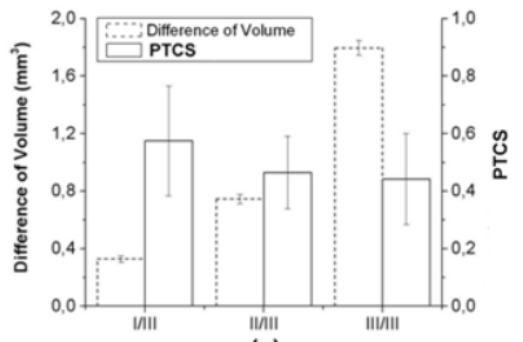

(c)

Figure 7. "Evaluating histograms". Histograms representing the total volume of dentin removed (dashed line) and the average of PTCS (solid line) for three levels (apical -a, middle -b and coronal -c) of each canal.

The software has to be improved to allow automatic characterization of canal branches. The presence of canal branches can require recursive refinement of the analysis to obtain quantitative evaluation of the interesting parameters. The co-registration procedure is automatic and simple; the user needs a short training period to learn how to manage the output produced by the algorithm. Images of canal cross-section in Figure 3 are produced by the software and can be used to evaluate if further improvement of the co-registration is needed. Data plotted in Figure 4 are available as text files for further statistical analysis not included in this software package.

Future work can be done to investigate a possible correlation between the volume of dentin removed and PTCS. Indeed from plots obtained from three different anatomical levels (see Figure 8), we can observe that these quantities are not independent. Of note, for the apical level (which is characterized by smaller canal diameter) the slope between volume of dentin removed and PTCS is higher than for the coronal level (the higher is the diameter of the native canal, the lower is the slope). This relationship could be an index to evaluate the quality of the endodontic treatment. Indeed the higher is the 
slope of the plot of Figure 8, the better will be the treatment quality, suggesting that less dentin was removed to obtain higher values of PTCS. New efforts have to be applied to push this method beyond research context to clinical practice, for example to evaluate quality of therapeutic intervention assessed by CBCT.

Figure 8. "Linking PTCS and volume of dentine removed". Linear regression between PTCS and volume of dentine removed in each different anatomical level: the slope of the fitted line is larger in the apical level (data are represented with black squares) and is smaller in the coronal level (blue triangles). Red circles correspond to data relative to the middle level of each canal.

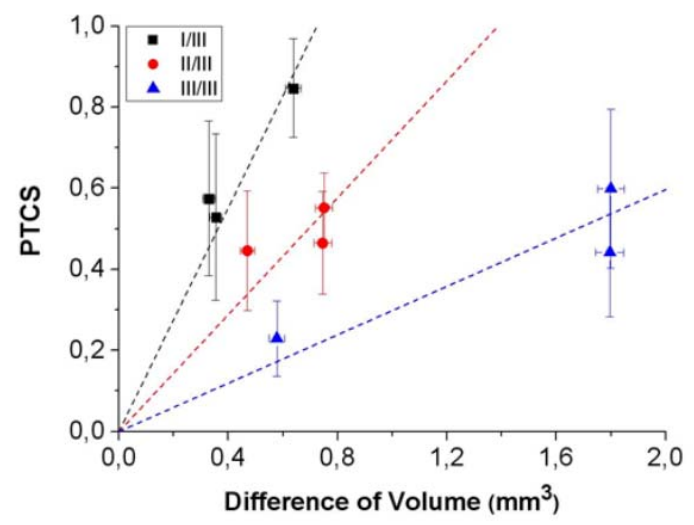

\subsection{Conservative treatment}

Composite filling is a widespread tooth restoration technique and the procedure is based on successive steps, which include bonding and placement of composite layer by layer. Incorrect handling of bonding procedure leads to formation of bulk bubbles in the composite and gaps at the dentin composite interface. Defects due to polymerization shrinkage of dental composite and different physical properties between tooth structure and restorative material are considered to be the most important factors in the formation of micro-leakage at the dentin-composite interface ${ }^{[44]}$. In this study the coregistration method adopted allowed obtaining some more information about the effect of the shrinkage on the native tooth structure.

Analysis of restoration quality and quantification of micro-leakage is traditionally done by cutting the tooth in slices for subsequent optical microscopy examination ${ }^{[4,}{ }^{46]}$. More recently some authors performed $\mu \mathrm{CT}$ imaging of ex-vivo restored teeth exploiting the possibility to investigate defects (gaps), calculate their volumes and eventually choose the 2D cutting planes for further optical microscopy analysis ${ }^{[47,48]}$. The $3 \mathrm{D}$ structure of tooth restoration revealed by $\mu \mathrm{CT}$ images demonstrates that the estimated gaps size and the number of defects revealed in 3D by $\mu \mathrm{CT}$ are always higher than microscopy ${ }^{[48]}$. Our investigation method, adding co-registration for direct comparison of pre- and post-shrinkage tooth structure, increases the value of the $\mu \mathrm{CT}$ examination of restorative treatment. The novelty of this co-registration approach resides in the possibility to highlight pre- and post-shrinkage differences inside the 3D tooth structure. Using the proposed pipeline there is no need of high computational resources and after co-registration the data analysis is very quick and straightforward.

In spite of the difficulty in using some features of this improved software, the user can obtain colour labeling of differences between the pre- and post-shrinkage structure (see Figure 6). In the investigated sample this analysis evidenced three unexpected occurrences: crack formation inside the tooth native structure, spatial displacement of part of the tooth structure in respect of the native conformation and formation of small pores at the interface between composite and tooth partially embedded inside the native tooth structure. Additionally, the developed algorithm demonstrates that photopolymerization and subsequent shrinkage can modify the original tooth structure. The co-registration algorithm developed for this case is not analytical and the user needs some days of training to learn how to obtain the best superposition between common parts of the two investigated structures. Also for experienced user the refinement of the co-registration can need from five to 10 runs. 
Practical applications of this method range from dentistry to orthopaedics where it is often necessary to obtain specular reconstruction of post-oncologic and post-traumatic damaged anatomic districts. Moreover in the research context the same method can be applied to multimodal imaging ${ }^{[49]}$ improving the structure characterization. Limitations are computational time and experience needed to perform the analysis. We tried to reduce these limitations by the following strategy: the user can choose some ROIs in the 3D space, which are processed by the algorithm instead of the whole sample volume.

Systematic studies are required to quantify the correlation and incidence of the crack formation and the spatial displacement related to the use of different composite and adhesive. Also micromechanical studies can be conducted to quantify these phenomena and evaluate the $3 \mathrm{D}$ stress and strain distribution inside the structure revealed by $\mu \mathrm{CT}{ }^{[3,23]}$. The analysis can guide new strategies to minimize the occurrence of such events, which can compromise the tooth stability and reduce the lifetime of the restored tooth.

\section{Conclusion}

Our results demonstrate that combining the detailed 3D structure of a tooth obtained by $\mu \mathrm{CT}$ imaging with appropriate software it is possible to quantify differences in the root and canal morphology related to therapeutic treatments. Software has been developed and optimized to evaluate changes by comparing pre- and post-treatment structures of treated sites. It should be interesting also to develop other algorithms to calculate the minimum distance between tooth external surface and canal surface before and after endodontic treatment and estimate the increasing of root wall fragility with finite element simulation as well as fragility of the same restored tooth with or without internal crack. At this stage the software tools developed are able to quantify the buoyancy of two very different dental treatments, endodontic and conservative, posing the basis for future development and for virtual dental laboratory.

\section{Acknowledgement}

This work was partially funded by P.O. F.S.E. Abruzzo 2007-2013 and European Community's Seventh Framework Programme (FP7/2007-2013), grant agreement n HEALTH-F5-2008-200859 MEGMRI.

\section{References}

[1] Roggendorf M. J., Legner M., Ebert J., Fillery E., Frankeberger R, Friedman S. Micro-CT evaluation of residual material in canals filled with Activ GP or GuttaFlow following removal with NiTi instruments. Journal of International Endodontic. 2010; 43: 200-209.

http://onlinelibrary.wiley.com/doi/10.1111/j.1365-2591.2009.01659.x/abstract;jsessionid=350C2D7ADBA7FA9BA13262CAA1 61CA21.d04t04. PMid: 20158531. http://dx.doi.org/10.1111/j.1365-2591.2009.01659.x

[2] Bergmans L., Van Cleynenbreugel J., Wevers M., Lambrechts P. A methodology for quantitative evaluation of root canal instrumentation using microcomputed tomography. Int. Endod. J. 2001; 34:390-398.

http://onlinelibrary.wiley.com/doi/10.1046/j.1365-2591.2001.00413.x/abstract. PMid: 11482723.

http://dx.doi.org/10.1046/j.1365-2591.2001.00413.x

[3] Magne P. Efficient 3D finite element analysis of dental restorative procedures using micro-CT data. Dental Material 2007; 23:539-548. http://www.demajournal.com/article/S0109-5641(06)00090-X/abstract. PMid: 16730058. http://dx.doi.org/10.1016/j.dental.2006.03.013

[4] Meleo D., Baggi L., Di Girolamo M., Di Carlo F., Pecci R., Bedini R. Fixture-abutment connection surface and micro-gap measurements by 3D micro-tomographic technique analysis. Ann Ist Super Sanita. 2012; 48:53-58. PubMed PMID: 22456016. http://www.iss.it/binary/publ/cont/ANN_12_01_09.pdf. PMid:22456016

[5] Chackartchi T., Iezzi G., Goldstein M., Klinger A., Soskolne A., Piattelli A., et al. Sinus floor augmentation using large (1-2 mm) or small $(0.2-1 \mathrm{~mm})$ bovine bone mineral particles: a prospective, intra-individual controlled clinical, micro-computerized tomography and histomorphometric study. Clinical Oral Implants Research 2011; 22:473-480. 
http://onlinelibrary.wiley.com/doi/10.1111/j.1600-0501.2010.02032.x/abstract. PMid: 21087317. http://dx.doi.org/10.1111/j.1600-0501.2010.02032.x

[6] Ilgenstein B., Deyhle H., Jaquiery C., Kunz C., Stalder A., Stubinger S., et al. Combined micro computed tomography and histology study of bone augmentation and distraction osteogenesis. Proc. of SPIE 2012; 85060M. http://proceedings.spiedigitallibrary.org/proceeding.aspx?articleid=1384267. http://dx.doi.org/10.1117/12.929616

[7] Jung M., Lommel D., Klimek J. The imaging of root canal obturation using micro-CT. International Endodontic Journal 2005; 38:617-626. http://onlinelibrary.wiley.com/doi/10.1111/j.1365-2591.2005.00990.x/abstract. PMid: 16104975. http://dx.doi.org/10.1111/j.1365-2591.2005.00990.x

[8] Edberg E. L., Kroese-Deutman H. C., Shih C. K., Lemoine J. J., Liebschner M. A., Miller M. J., et al. Methods: a comparative study of radiography, microcomputed tomography, and histology for bone tissue engineering. Tissue Engineering 2005; 11:1356-1367. http://online.liebertpub.com/doi/abs/10.1089/ten.2005.11.1356. PMid: 16259591. http://dx.doi.org/10.1089/ten.2005.11.1356

[9] Sinibaldi R., Pecci R., Somma F., Della Penna S., Bedini R. A new software for dimensional measurements in 3D endodontic root canal instrumentation. Ann Ist Super Sanita. 2012; 48:42-48. http://www.iss.it/binary/publ/cont/ANN_12_01_07.pdf. PMid:22456014

[10] Plotino G., Grande N.M., Pecci R., Bedini R., Pameijer C.H., Somma F. Three-dimensional imaging using microcomputed tomography for studying tooth macromorphology, JADA 2006; 137, 1555-1561. http://jada.ada.org/content/137/11/1555.abstract . PMid:17082282

[11] Scarfe W. C., Farman A. G., Sukovic P. Clinical application of cone-beam computed tomography in dental practice. J. Can. Dent. Assoc. 2006; 72:75-80. http://www.cda-adc.ca/jcda/vol-72/issue-1/75.pdf. PMid:16480609

[12] Patel S., Dawood A., Whaites E., Pitt Ford T. The potential applications of cone-beam computed tomography in the management of endodontic problems. International Endodontic Journal 2007; 40:818-830.

http://onlinelibrary.wiley.com/doi/10.1111/j.1365-2591.2007.01299.x/abstract. PMid: 17697108. http://dx.doi.org/10.1111/j.1365-2591.2007.01299.x

[13] Ganz S. D. Cone beam computed tomography-assisted treatment planning concepts. Dent Clin North Am 2011; 55:515-536. http://www.sciencedirect.com/science/article/pii/S0011853211000334. PMid: 21726687. http://dx.doi.org/10.1016/j.cden.2011.02.019

[14] Kumar V., And D., Williams K., Walker P. M. Comparison of two cone beam computed tomography multiplanar reconstruction orientation protocols. Journal of Biomedical Graphics and Computing 2013; 3:7-15. http://www.sciedu.ca/journal/index.php/jbgc/article/view/1274. http://dx.doi.org/10.5430/jbgc.v3n2p7

[15] Sakellariou A., Arns C., Sheppard A., Sok R., Averdunk H., Limaye A., et al. Developing a virtual materials laboratory. Materialstoday 2007; 510:44-51. http://www.sciencedirect.com/science/article/pii/S1369702107703073. http://dx.doi.org/10.1016/S1369-7021(07)70307-3

[16] Rossella Bedini, Deborah Meleo, Raffaella Pecci. Microtomography and Its Application in Oral and Implant Research D. Iacoviello and U. Andreaus (eds.), Biomedical Imaging and Computational Modeling in Biomechanics, Lecture Notes in Computational Vision and Biomechanics 4, DOI 10.1007/978-94-007-4270-3_8, \# Springer Science+Business Media Dordrecht 2013. http://link.springer.com/chapter/10.1007\%2F978-94-007-4270-3_8

[17] Pozdnyakova A., Giuliani A., Dutkiewiczz J., Babutsky A., Chyzhyk A., Roether J.A., et al. Analysis of porosity in NiTi SMA's changed by secondary pulse electric current treatment by means of Ultra Small Angle Scattering and micro-computed tomography. Intermetallics 2010; 18:907-912. http://www.sciencedirect.com/science/article/pii/S0966979509003458. http://dx.doi.org/10.1016/j.intermet.2009.12.030

[18] Komlev V.S., Mastrogiacomo M., Pereira R. C., Peyrin F., Rustichelli F., Cancedda R. Biodegradation of porous calcium phosphate scaffolds in an ectopic bone formation model studied by X-ray computed micro-tomography 2010; 19:136-146. http://www.ecmjournal.org/journal/papers/vol019/vol019a14.php

[19] Ovaysi S., Piri M. Direct pore-level modelling of incompressible fluid flow in porous media. Journal of Computational Physics 2010; 229: 7456-7476. http://www.sciencedirect.com/science/article/pii/S0021999110003438. http://dx.doi.org/10.1016/j.jcp.2010.06.028

[20] De Santis R., Mollica F., Prisco F., Rengo S., Ambrosio L., Nicolais L. A 3d analysis of mechanically stressed dentin-adhesive-composite interfaces using x-ray micro-ct. Biomaterials 2005; 26:257-270.

http://www.sciencedirect.com/science/article/pii/S0142961204001632. PMid: 15262468. http://dx.doi.org/10.1016/j.biomaterials.2004.02.024

[21] Contardo L., De Luca M., Biasotto M., Longo R., Olivo A., Pani S., et al. Evaluation of the endodontic apical seal after post insertion by synchrotron radiation microtomography. Nuclear Instruments and Methods in Physics Research Section A: 
Accelerators, Spectrometers, Detectors and Associated Equipment 2005; 548:253-256. http://eprints.ucl.ac.uk/116794/. http://dx.doi.org/10.1016/j.nima.2005.03.098

[22] Scheiner S., Sinibaldi R., Pichler B., Komlev V., Renghini C., Vitale-Brovarone C., et al. Micromechanics of bone tissue-engineering scaffolds, based on resolution error-cleared computer tomography. Biomaterials. 2009; 30:2411-2419. http://www.sciencedirect.com/science/article/pii/S014296120801051X. PMid: 19135717. http://dx.doi.org/10.1016/j.biomaterials.2008.12.048

[23] Dejaco A., Komlev V. S., Jaroszewicz J., Swiezkowski W., Hellmich C. Micro CT-based multiscale elasticity of double- porous (pre-cracked) hydroxyapatite granules for regenerative medicine. Journal of Biomechanics 2012; 45:1068-1075. http://www.sciencedirect.com/science/article/pii/S0021929012000279. PMid: 22296936. http://dx.doi.org/10.1016/j.jbiomech.2011.12.026

[24] Dowker S., Davis G., Elliott J. X-ray microtomography: Nondestructive threedimensional imaging for in vitro endodontic studies. Oral Surgery, Oral Medicine, Oral Pathology, Oral Radiology and Endodontology 1997; 83:510-516. http://www.sciencedirect.com/science/article/pii/S1079210497901554

[25] Gao Y., Peters O. A., Wu H., Zhou X. An application framework of three-dimensional reconstruction and measurements for endodontic research. Journal of Endodontics 2009; 35:269-274. http://dental.pacific.edu/Documents/faculty_research/labs/endo/JOE200902.pdf. PMid: 19166788. http://dx.doi.org/10.1016/j.joen.2008.11.011

[26] Paque F., Barbakow F., Peters O. A. Root canal preparation with Endo-Eze AET: changes in root canal shape assessed by micro-computed tomography. International Endodontic Journal 2005; 38:456-464.

http://onlinelibrary.wiley.com/doi/10.1111/j.1365-2591.2005.00968.x/abstract. PMid: 15946266. http://dx.doi.org/10.1111/j.1365-2591.2005.00968.x

[27] Salome M., Peyrin F., Cloetens P., Odet C., Laval-Jeantet A.M., Baruchel J., et al. A synchrotron radiation microtomography system for the analysis of trabecular bone samples. Med Phys 1999; 26: 2194-2204. http://online.medphys.org/resource/1/mphya6/v26/110/p2194_s1?isAuthorized=no. PMid: 10535638. http://dx.doi.org/10.1118/1.598736

[28] Nikhil R., Sankar K. A review on image segmentation techniques. Pattern Recognition 1993; 26:1277-1294. http://www.sciencedirect.com/science/article/pii/003132039390135J. http://dx.doi.org/10.1016/0031-3203(93)90135-J

[29] Zhu S. C. Region competition: Unifying snakes, region growing, and bayes/mdl for multiband image segmentation. IEEE Transactions on Pattern Analysis and Machine Intelligence 1996; 18:884-900. http://ieeexplore.ieee.org/xpl/login.jsp?tp=\&arnumber=466909\&url=http\%3A\%2F\%2Fieeexplore.ieee.org\%2Fxpls\%2Fabs_all.j sp\%3Farnumber\%3D466909. http://dx.doi.org/10.1109/34.537343

[30] Goldstein H. Classical Mechanics. Addison-Wesley, Reading, MA 2001. http://books.google.it/books/about/Classical_mechanics.html?id=W_YgAAAAMAAJ\&redir_esc=y

[31] Maes F., Collignon A., Vandermeulen D., Marchal G., Suetens P. Multimodality image registration by maximization of mutual information. IEEE Transactions on Medical Imaging 1997; 16:187-198.

http://ieexplore.ieee.org/xpl/login.jsp?tp=\&arnumber=563664\&url=http\%3A\%2F\%2Fieexplore.iee.org\%2Fiel1\%2F42\%2F1 2249\%2F00563664. PMid: 9101328. http://dx.doi.org/10.1109/42.563664

[32] Ingber L. Adaptive simulated annealing (ASA): Lessons learned. Control and Cybernetics 1996; 25:33-54. http://www.ingber.com/asa96_lessons.pdf

[33] Studholme C., Hill D. L. G., Hawkes D. J. An overlap invariant entropy measure of 3D medical image alignment. Pattern Recognition 1999; 32:71-86. http://eecs.vanderbilt.edu/courses/cs359/other_links/papers/studholme_NMI_1999.pdf. http://dx.doi.org/10.1016/S0031-3203(98)00091-0

[34] MacKay D. J. Information Theory, Inference, and Learning Algorithms. Cambridge University Press, 2005.

[35] Collignon A., Maes F., Delaere D., Vandermeulen D., Suetens P., Marchal G. "Automated multimodality medical image registration using information theory," in Proc. 14th Int. Conf. Information Processing in Medical Imaging; Computational Imaging and Vision 3: June 1995; Ile de Berder. Edited by Bizais Y., Barillot C., Di Paola R.: Kluwer, 1995: $263-274$. http://citeseerx.ist.psu.edu/viewdoc/summary?doi=10.1.1.85.1157

[36] Viola P., Wells III W. M. Alignment by maximization of mutual information. International Journal of Computer Vision, 1997: 24, $137-154$. http://ieeexplore.ieee.org/xpl/login.jsp?tp=\&arnumber=466930\&url=http\%3A\%2F\%2Fieeexplore.ieee.org\%2Fxpls\%2Fabs_all.j sp\%3Farnumber\%3D466930. http://dx.doi.org/10.1023/A:1007958904918

[37] Pioch T., Stotz S., Buff E., Duschner H., Staehle H.J. Influence of different etching times on hybrid layer formation and tensile bond strength. Am J Dent 1998; 11:202-206. 
http://pubget.com/paper/10388377/Influence_of_different_etching_times_on_hybrid_layer_formation_and_tensile_bond_strengt h. PMid:10388377

[38] Hubscher W., Barbakow F., Peters O.A. Root-canal preparation with Flex-Master: canal shapes analysed by micro-computed tomography. Int Endod J 2003; 36:740-747. http://onlinelibrary.wiley.com/doi/10.1046/j.1365-2591.2003.00723.x/abstract. PMid: 14641437. http://dx.doi.org/10.1046/j.1365-2591.2003.00723.x

[39] Vertucci F. J. Root canal morphology and ts relationship to endodontic procedures. Endodontic Topics 2005; 10:3-29. http://onlinelibrary.wiley.com/doi/10.1111/j.1601-1546.2005.00129.x/abstract?deniedAccessCustomisedMessage=\&userIsAuthe nticated=false. http://dx.doi.org/10.1111/j.1601-1546.2005.00129.x

[40] Somma F, Cretella G, Carotenuto M, Pecci R, Bedini R, De Biasi M, et al. Quality of thermoplasticized and single point root fillings assessed by micro-computed tomography. Int Endod J. 2011; 44:362-369. http://onlinelibrary.wiley.com/doi/10.1111/j.1365-2591.2010.01840.x/abstract. PMid: 21255040. http://dx.doi.org/10.1111/j.1365-2591.2010.01840.x

[41] Villette G., Manek S., Legner M., Fillery E. D., Torneck C. D., Basrani B. R., et al. Characterization of an ex vivo model for the assessment of root canal disinfection. Journal of Endodontics 2008; 34:1490-1496. http://www.ncbi.nlm.nih.gov/pubmed/19026880. PMid: 19026880. http://dx.doi.org/10.1016/j.joen.2008.08.038

[42] Dugas N. N., Lawrence H. P., Teplitsky P., Friedman S. Quality of life and satisfaction outcomes of endodontic treatment. Journal of Endodontics 2002; 28:819-827. http://www.jendodon.com/article/S0099-2399(05)60776-5/abstract. PMid: 12489651. http://dx.doi.org/10.1097/00004770-200212000-00007

[43] Zaslansky P., Fratzl P., Rack A., Wu M-K., Wesselink P.R., Shemesh H. Identification of root filling interfaces by microscopy and tomography methods. International Endodontic Journal 2011; 44:395-401. http://onlinelibrary.wiley.com/doi/10.1111/j.1365-2591.2010.01830.x/pdf. PMid: 21219359. http://dx.doi.org/10.1111/j.1365-2591.2010.01830.x

[44] Retief D. H. Do adhesives prevent microleakage? Int. Dent. J. 1994; 44:19-26. PMid:8021028

[45] Opdam N. J. M., Roeters J. J. M., Joosten M., Veeke, O. V. Porosities and voids in class I restorations placed by six operators using a packable or syringable composite. Dental Materials 2002; 18: 58-63.

http://www.demajournal.com/article/S0109-5641(01)00020-3/abstract. http://dx.doi.org/10.1016/S0109-5641(01)00020-3

[46] Samet N., Kwon K.R., Good P., Weber H.P. Voids and interlayer gaps in class 1 posterior composite restorations: a comparison between a microlayer and a 2-layer technique. Quinteessence International, 2006; 37: 803-809. http://qi.quintessenz.de/index.php?doc=abstract\&abstractID=11517/

[47] Sun J., Eidelman N., Lin-Gibson S. 3D mapping of polymerization shrinkage using X-ray micro-computed tomography to predict microleakeage. Dental Material 2009; 25:314-320. http://www.sciencedirect.com/science/article/pii/S0109564108002017. PMid: 18762335. http://dx.doi.org/10.1016/j.dental.2008.07.010

[48] Deyhle H., Schmidli F., Krastl G., Muller B. Evaluating tooth restorations: Micro computed tomography in practical training for students in dentistry. Proc. of SPIE 2010; 780417:1-9.

http:/www.deepdyve.com/lp/spie/evaluating-tooth-restorations-micro-computed-tomography-in-practical-kb02zF6dhZ

[49] Mouchess M. L., Sohara Y., Nelson M. D. Jr., DeClerck Y. A., Moats R. A. Multimodal imaging analysis of tumor progression and bone resorption in a murine cancer mode. Journal of Computer assisted tomography 2006; 30:525-534.

http://journals.lww.com/jcat/Abstract/2006/05000/Multimodal_Imaging_Analysis_of_Tumor_Progression.30.aspx. PMid: 16778634. http://dx.doi.org/10.1097/00004728-200605000-00030 